\title{
Metodologia e Software Educacional para a Investigação e Remediação de Erros Conceituais em Matemática
}

Title: Methodology and Educational Software for Investigating and Remediating Errors in Mathematics

\author{
Diego Marczal \\ Coordenação do Curso de Tecnologia \\ em Sistemas para Internet \\ Universidade Tecnológica Federal do \\ Paraná (UTFPR) \\ Guarapuava, Paraná, Brasil \\ marczal@utfpr.edu.br
}

\author{
Alexandre Ibrahim Direne \\ Departamento de Informática \\ Universidade Federal do Paraná \\ (UFPR) \\ Curitiba, Paraná, Brasil \\ alexd@inf.ufpr.br
}

\author{
Andrey Ricardo Pimentel \\ Departamento de Informática \\ Universidade Federal do Paraná \\ (UFPR) \\ Curitiba, Paraná, Brasil \\ andrey@inf.ufpr.br
}

Eleandro Maschio

Coordenação do Curso de Tecnologia em Sistemas para Internet

Universidade Tecnológica Federal do Paraná (UTFPR)

Guarapuava, Paraná, Brasil

eleandrom@utfpr.edu.br

\author{
Ana Carla Borille \\ Departamento de Informática \\ Universidade Federal do Paraná \\ (UFPR) \\ Curitiba, Paraná, Brasil \\ carlaborille@gmail.com
}

\begin{abstract}
Resumo A aprendizagem de Matemática tem intrigado pesquisadores e educadores há muito tempo, principalmente no que se refere a erros conceituais. Apesar dos avanços das tecnologias digitais no tratamento de erros da aprendizagem, poucos resultados concretos foram atingidos. Mais recentemente, os institutos oficiais de avaliação escolar do Brasil apontaram limitações no aprendizado do Matemática, principalmente no ensino médio. Assim, a criação de métodos inovadores e ferramentas de software de apoio pedagógico que ajudem tanto o aprendiz como o professor a revisitarem os erros cometidos passa a ser uma perspectiva relevante de pesquisa. Este artigo apresenta um método e uma ferramenta web (FARMA) de autoria e aprendizagem com enfoque nas ideias de retroação a contextos de erro e mobilidade tecnológico-educacional. A metáfora de retroação ao contexto de um erro do aprendiz se constitui na abordagem cognitivista do projeto e implementação da FARMA. Com ela, aprendizes e professores podem se comunicar dentro e fora da sala de aula para analisar e entender a natureza dos erros. Resultados experimentais mostram que há beneficios educacionais estatisticamente significativos com a utilização do método de autoria de problemas aqui proposto, assim como a solução dos mesmos, por meio da FARMA.
\end{abstract}

Palavras-Chave: Retroação a erros, Remediação a erros, Ambientes de autoria, Objetos de aprendizagem. 


\begin{abstract}
Maths learning has intrigued researchers and educator for a long time, especially in what relates to buggy concepts. Despite the advances in digital technologies aimed at treating learning errors, few concrete results have been accomplished. More recently, official Brazilian institutions that evaluate school performance concluded that maths learning is insufficient in high school level. Thus, the development of innovating methods and software tools for pedagogic support that help both teaches and students in revisiting errors is a relevant research perspective. The paper presents a new method and a web-based, authoring-learning tool (FARMA) that focuses on the ideas of learner-error context backtracking as well as of tech-educational mobility. The metaphor behind learner-error context backtracking consists of the cognitive approach that guided the design and implementation of FARMA. With it, teachers and students can communicate in and out of the classroom for analyzing and understanding the nature of an error. Experimental results show that there are statistically relevant educational benefits in applying the proposed method in authoring problems statements as well as the solution of such problems through FARMA
\end{abstract}

Keywords: Retroaction of errors, Error feedback, Authoring environments, Learning objects 


\section{Introdução}

A educação no Brasil é constantemente avaliada por exames de larga escala, como por exemplo, o Instituto Nacional de Estudos e Pesquisas Educacionais "Anísio Teixeira" (INEP)1, Exame Nacional do Ensino Médio (ENEM), Exame Nacional de Desempenho dos Estudantes (ENADE).

O INEP tem como objetivo principal realizar o levantamento e a classificação do nível de conhecimento dos estudantes das instituições públicas de ensino do país, seus resultados são utilizados como base para a melhoria da qualidade de ensino e aprendizagem. Dentre as avaliações realizadas, a Avaliação Nacional da Educação Básica (Aneb) é a única que abrange o Ensino Fundamental e Médio, sendo aplicada em áreas da Língua Portuguesa, com foco em interpretação de texto, e Matemática, centrada na resolução de problemas.

$\mathrm{Na}$ área da Matemática (foco do trabalho), a prova da Aneb possui escalas de avaliação para o Ensino Fundamental e Médio. As escalas de avaliação do Ensino Fundamental começam no nível 0 (zero) e terminam no nível 12 (doze). Para o Ensino Médio, as escalas variam de 0 (zero) a 4 (quatro), em que 0 é o nível mais baixo de conhecimento e o 4 mais alto.

Da avaliação realizada em 2011 para área da Matemática2, em âmbito nacional, o nível alcançado pelos anos iniciais do Ensino Fundamental foi 4 e pelos anos finais foi 6, de 13 níveis ( 0 a 12). Já para o Ensino Médio, o resultado atingido pelo $3^{\circ}$ (terceiro) ano/série foi 0 de 5 níveis (0 a 4). Na última avaliação, realizada em 20133, em âmbito nacional, os níveis se mantiveram.

Esses resultados indicam que existe uma grande deficiência no ensino e aprendizagem da Matemática, pois os aprendizes sequer alcançaram $50 \%$ dos níveis avaliados. Com isso, destaca-se a importância da busca por novas abordagens de ensino e de aprendizagem da Matemática, além do aperfeiçoamento das já existentes. Outras pesquisas, como a de Flores [1], também destacam dificuldades no ensino e aprendizado de Matemática por meio de resultados de avaliações nacionais.

Segundo estudos de Notare e Behar [2], os professores conhecem as dificuldades dos aprendizes no ensino da Matemática e constantemente buscam novas alternativas pedagógicas. Porém, ainda existem grandes objeções em alterar as metodologias tradicionais de ensino. Isso evidencia a necessidade do desenvolvimento de novas

\footnotetext{
${ }^{1}$ Disponível em: http://portal.inep.gov.br

${ }^{2}$ Resultados Aneb 2011:

http://sistemasprovabrasil2.inep.gov.br/resultados

${ }^{3}$ Resultados Aneb 2013:

$\mathrm{ftp}$ //ftp.inep.gov.br/microdados/microdados_aneb_prova_brasil_2013.zip
}

abordagens metodológicas para o ensino e aprendizagem da Matemática. Mas para que uma pedagogia mais centrada na aprendizagem realmente traga experiências de sucesso, deve-se ainda apoiar o professor como elemento chave na construção de currículos e geração de conteúdos.

Nesse sentido, uma das abordagens de maior destaque é o uso das tecnologias digitais no processo de ensinoaprendizagem. No que diz respeito à Matemática, essas tecnologias ajudam o aprendiz principalmente por meio da simulação computacional, em que situações do mundo real permitem a visualização de conceitos por meio de uma representação intuitiva.

Porém, a criação de softwares Educacionais, como Sistemas Tutores Inteligentes (STIs) e os Objetos de Aprendizagem (OAs), que promovam intuitividade nas simulações não é uma tarefa simples. Murrray [3] observou 20 ferramentas de autoria destacando que elas são complexas, sendo difícil imaginar seu uso, por professores, sem um suporte contínuo. Ainda, segundo Murray, a produção de um STI que ocupe o tempo de 1 hora em sala de aula, exige um esforço de 300 a 1.000 horas de trabalho de autoria.

Pesquisas tentaram minimizar esse esforço, sendo que as mais bem sucedidas foram aquelas que propuseram ferramentas de autoria como mecanismos centrais de seus resultados científicos. No entanto, tais ferramentas ainda são de difícil acesso e compreensão por parte de muitos autores (professores) de conteúdos [4].

Em paralelo, a web constitui um dos meios mais importantes para a disponibilização e execução de OAs, além de oferecer recursos para individualizar o perfil de aprendizes e monitorá-los por longos períodos de tempo. Com esse crescimento, fica evidente a necessidade de ferramentas de autoria totalmente voltadas para a web, pois com elas o professor pode construir softwares educacionais e disponibilizá-los ao aprendiz de forma a promover até mesmo o seu uso sob aspectos de mobilidade tecnológico-educacional [5].

Em contrapartida, durante os estudos, o aprendiz pode errar por descuido ou pela falta de conhecimento de conceitos. No segundo caso, a remediação do erro pode ser demorada e até depender da ajuda do professor. Consequentemente, uma nova tentativa de resolver o mesmo exercício poderá exigir algum tempo (talvez várias semanas), pois dependerá de uma reestruturação cognitiva do aprendiz. Por isso, o erro no processo de aprendizagem não pode ser negligenciado, mesmo em OAs construídos por professores com pouco conhecimento em programação.

Assim, o objetivo deste trabalho foi a pesquisa, desenvolvimento e a aplicação de uma ferramenta de auto- 
ria web com foco na construção de OAs destinados ao ensino de conceitos matemáticos. Buscou-se possibilitar que o erro cometido pelo aprendiz fosse utilizado como uma oportunidade de aprendizagem, isso por meio de mecanismos que permitissem remediação e retroação (ver detalhes na Subseção 3.2) a erros a curto e longo prazo. Adicionalmente, que possa ser utilizada por professores com conhecimentos básicos em informática.

Para avaliar a ferramenta proposta, e principalmente seu mecanismo de retroação a erros (diferencial da pesquisa), foram realizados experimentos com alunos e professores de escolas públicas. Os resultados indicaram que a ferramenta teve uma boa aceitação por parte dos professores e que os alunos que interagiram com os OAs construídos com ela e fizeram uso do mecanismo de retroação a erros apresentaram um aumento significativo na aquisição de conhecimento quando comparados com os que não usaram o mecanismo de retroação.

\section{Resenha Literária}

Os Sistemas Tutores Inteligentes (STIs) são ferramentas educativas que têm por objetivo assistir aprendizes e professores no processo de aquisição e transmissão de conhecimento. Uma das características de destaque dos STIs é a possibilidade de trabalhar com os erros dos aprendizes. A partir desses erros, os STIs podem identificar as dificuldades individuais do aprendiz e então fornecer um feedback apropriado para corrigir desvios na formação de conceitos [6]. Tais ações são baseadas em teorias de aprendizagem, como aquelas propostas pelo ACT (Adaptive Control of Thought), que se fundamentam em manter o aprendiz em uma linha de aprendizagem ideal, mesmo que restrita [7].

O Geometry Tutor de Anderson [6] é um exemplo de STI para ensino da geometria que explorou os erros dos aprendizes por meio de um módulo de diagnóstico automático de erros. O conhecimento do sistema tutor é fundamentado em regras de produção, sendo que não possui modelagem dinâmica do aprendiz, porém utiliza um modelo ideal que representa todo o conhecimento que supostamente o aprendiz deve adquirir.

Outro STI é o PAT2Math [8], voltado para o ensino de álgebra elementar, com equações de $1^{\circ}$ e $2^{\circ}$ graus de uma incógnita. Seu principal componente é o PATequation, um módulo de resolução de equações que permite ao aluno resolver uma equação passo a passo. $\mathrm{O}$ módulo provê ao aluno a correção e feedback em tempo real de cada passo fornecido. Apesar de ter apresentado sucesso em suas pesquisas, o sistema não guarda nem monitora os erros para mostrá-los aos aprendizes e tutores a longo prazo.
As teorias da ACT, apesar de bem sucedidas em STIs, limitam-se a condições em que o feedback é explícito, imediato e em que os erros do aprendiz possam ser inteiramente identificados. Além disso, existem poucas pesquisas sobre seu uso em micromundos e objetos de aprendizagem, principalmente sobre ferramentas de autoria para gerar material capaz de registrar e monitorar tais abordagens dos erros.

\subsection{Ambientes de Autoria para STIs}

Autoria de material digital, ou simplesmente autoria, em Educação, é o processo de organizar e/ou produzir uma sequência de informações de modo a compor um material para o ensino-aprendizagem, (p. ex., STIs e OAs) que possibilite a interação do usuário com partes de um domínio específico. Com isso, pode-se definir uma ferramenta de autoria como um software em que um tutor humano possa manipular, criar, alterar ou excluir sessões de ensino.

No passado, pesquisas foram realizadas nesta área para o desenvolvimento de ferramentas de autoria para STIs [6]. Um exemplo recente de ferramenta de autoria, o EDUCA, permite a construção de conteúdos educacionais personalizados que podem ser utilizados de maneira colaborativa. Em seu trabalho, Cabada et al. [9] propõem uma abordagem para determinar o estilo de aprendizado do indivíduo usando redes de mapas auto-organizáveis (Kohonen neural networks). A rede é atualizada por questionários fornecidos ao aprendiz, durante a interação com o conteúdo.

O EDUCA apresenta características interessantes, como o aprendizado colaborativo mobile, além da definição do estilo do aprendiz para recomendação de material de autoria. No entanto, há relatos de avaliação do sistema apenas quanto à ferramenta de autoria, e não quanto ao processo de aprendizagem. Além disso, não há descrição do modo como os erros dos aprendizes podem ajudar na recomendação dos conteúdos e consequentemente no processo de aprendizagem.

O CTAT (Cognitive Tutor Authoring Tools) [10] é um projeto com o objetivo de prover um conjunto de ferramentas de autoria para o desenvolvimento de dois tipos principais de STIs: cognitivos e baseados em exemplos. O primeiro exige autores com conhecimento em programação e inteligência artificial. Já o segundo, com os mesmos recursos do primeiro, não requer nenhum conhecimento em programação de computadores.

Já o IGeom [11] é um software para Geometria Dinâmica (GD) que permite realizar todas as operações básicas da Geometria. Um dos módulos oferecidos por essa ferramenta permite a autoria de exercícios envolven- 
do a GD, assim como a correção automática pelo sistema. $\mathrm{O}$ uso do CTAT permite que, durante a resolução de um problema, o tutor possa identificar os passos do aprendiz e lançar algum tipo de feedback ou sugestão. Apesar do sistema oferecer feedback imediato sobre os erros dos aprendizes, não destaca a exploração e retroação ao contexto de erro, nem pelo professor nem pelo aprendiz.

\subsection{Ambientes de Autoria para Objetos de Aprendizagem}

Os OAs também podem ser vistos como um recurso cognitivo para auxiliar e ampliar o ensino e aprendizagem com características peculiares, dentre as quais destacamse a reusabilidade, granularidade, acessibilidade e interoperabilidade. No quesito softwares de autoria para OAs, pode-se evidenciar algumas ferramentas como o CourseLab, eXe Learning, HotPotatoes, Microsoft LCDS e MyUdutu, as quais foram destacadas e avaliadas em pesquisas anteriores apresentadas por Battistella e Von Wangenheim [12].

Todas essas ferramentas são bem sucedidas para a produção de arquivos digitais em diferentes mídias, tais como texto, imagem, som e vídeo. Entretanto, os objetos criados a partir dessas ferramentas fornecem pouca interação com o aprendiz, principalmente no que se refere ao sentido crítico da carência de recursos de avaliação formativa, atuando apenas na simples avaliação somativa. Em particular, os ambientes citados não tratam explicitamente os erros cometidos pelo aprendiz como uma importante fonte de apoio ao aprendizado autônomo. Uma tentativa de trabalhar com erro em ferramentas de autoria para Objetos de Aprendizagem foi apresentada por Stamey e Saunders [13], mas não promove condições de exploração dos erros dos aprendizes, apenas opções de reestruturação da questão.

Nessa direção, também pode-se destacar a aprendizagem sócio-interacionista proposta por Vygotski [14]. Ela propõe que a compreensão do ser humano acontece a partir de sua cultura e interação com os indivíduos, na Educação, tem objetivo de estimular o desenvolvimento cognitivo do aprendiz para obter um conhecimento sólido e aprofundado.

A concepção da aprendizagem sócio-interacionista valoriza situações didáticas nas quais os alunos devem explicitar seus procedimentos de resolução e argumentar sobre suas estratégias. Nesta pesquisa, há o destaque para a teoria sócio-interacionista no sentido de o aprendiz ter a oportunidade de rever, analisar, refletir e até mesmo refazer atividades em que cometeu equívocos durante sua interação.

\section{Fundamentos da Solução}

Uma das características mais importantes na construção de sistemas para o amparo do ensino está no desenvolvimento do conteúdo, pois a dificuldade na sua elaboração pode ser um fator determinante na utilização ou não de uma ferramenta. Por isso, um ambiente de autoria, além de permitir criar conteúdos de qualidade, interativos e que produzam uma avaliação formativa, devem também oferecer: (a) a diminuição dos esforços necessários para a construção de softwares educacionais; (b) a diminuição da habilidade mínima necessária para lidar com conteúdos fora do domínio específico do autor; (c) a facilidade de prototipagem rápida. Em conjunto, tais objetivos devem proporcionar uma forma de ensino e aprendizagem progressivamente mais eficiente no sentido de elaboração e aplicação de softwares educacionais [6].

Por outro lado, em uma sessão de estudo, o aprendiz está sujeito a erros, seja por descuido ou por falta de conhecimento de conceitos. Assim, é importante se beneficiar deste fato para promover a construção do conhecimento do aprendiz. Por meio do erro, pode-se oferecer duas principais formas de aprendizado: a remediação de erros, na qual o aprendiz recebe um feedback imediato sobre a falha, permitindo a sua recuperação e a continuação dos estudos; e a retroação aos erros, em que o aprendiz pode explorá-los e, a partir disso, tentar refazer a interação onde o erro foi cometido para entender seu motivo e então solucioná-lo.

\subsection{O Contexto de Erros}

Presente na trajetória escolar, o erro ocorre independentemente de idade e/ou de nível de desempenho, sendo uma das etapas fundamentais do processo de aprendizagem. O erro foi, muitas vezes, relacionado apenas aos aspectos negativos do processo de aprendizagem. Devido à sua natureza, o erro não fornece apenas dados sobre as falhas do aprendiz, quando analisado também pode identificar suas reais necessidades e então oferecer subsídios para abordagens de ensino diferenciadas, de modo a suprir as dificuldades encontradas pelo aprendiz. Por essa razão, entre outras, o erro cada vez mais está sendo foco de pesquisas, considerando-o uma etapa natural na construção do conhecimento $[15,16]$.

O erro, no arcabouço proposto, é considerado um desvio da solução correta de um exercício (ou questão de um exercício), sendo que eles ocorrem durante a resolução das questões de cada exercício proposto pelo professor. Este pode ter as mais variadas causas como simples enganos, equívocos ou demonstrações de desconhecimento sobre o assunto abordado. Por meio de seus mecanismos, o arcabouço proposto destaca que o erro deve ser 
interpretado como uma evidência do estágio de desenvolvimento do aprendiz, e portanto deve ser encarado pelo professor como um momento do processo de construção da aprendizagem, buscando auxiliar o aprendiz na sua superação e então alcançar os objetivos propostos.

Para isso, é necessário registrar todas as informações relevantes sobre os erros do aprendiz, desde a hora exata da ocorrência do erro, assim como todo o contexto onde ele aconteceu (como o exercício, a questão, o número de tentativas, as dicas recebidas, etc.). Isso possibilita que o professor possa voltar ao momento exato da ocorrência do erro, recuperando todo seu contexto e visualizando de forma clara o que o aprendiz visualizava no momento da ocorrência. A partir disso, pode-se analisar e propor soluções alternativas ao aprendiz mesmo após um longo período de tempo da ocorrência do erro. Da mesma forma, o aprendiz também pode recuperar o contexto do seu erro e então fazer análises e refazer o exercício do ponto em que ele errou. Isso também promove um aprendizado individual, em que o erro se torna um processo de maturidade e oportunidade para o aprendiz desenvolver sua capacidade de pensar e resolver situações-problema, criar hipóteses e assim chegar a um novo conhecimento, alcançando um objetivo final.

\subsection{Remediação por Retroação}

Como mencionado, durante seus estudos, o aprendiz pode errar por descuido ou pela falta de conhecimento de algum conceito. No segundo caso, a remediação do erro pode ser demorada e até depender da ajuda do professor. Consequentemente, uma nova tentativa de resolver o mesmo exercício poderá exigir algum tempo (talvez várias semanas) pois dependerá de uma reestruturação cognitiva do aprendiz. No entanto, com a possibilidade de o aprendiz retroagir ao contexto do erro de maneira autônoma (que só depende da interação com a FARMA), é possível alcançar uma dinâmica de auto estudo fundamentalmente distinta das existentes nos OAs construídos até hoje.

Tal comportamento do aprendiz só é possível se implementado um mecanismo de modelagem de longo prazo do aprendiz, acoplado à base de exercícios criados pelo autor do material eletrônico. Com isso, o erro deixa de ser apenas uma forma de avaliar somativamente o usuário para se tornar a modalidade concreta de avaliação formativa na construção do conhecimento, tal como advogou a psicologia cognitivista de Piaget, Papert e seus seguidores. Em outras palavras, a alternância entre a realização de novos exercícios e a restauração do contexto de antigos enunciados que foram resolvidos com erro parece se alinhar com as visões de futuro que estão nas mais promissoras perspectivas de mudança pedagógica qualitativamente relevante.

Alguns estudos como os de Isotani et al. [17] e McLaren et al. [18] usam os erros para prover o aprendizado de outra maneira em que, ao invés de esperar o erro do aprendiz, apresentam-se exemplos incorretos para que os aprendizes construam seu conhecimento a partir deles. Em seus artigos, eles destacam que existem evidências para que o estudo a partir de exemplos incorretos pode ser útil para o aprendizado, especialmente a longo prazo, quando o estudante já teve a oportunidade de refletir sobre suas ações.

Nesta linha, Adams et. al. [19], apresentam um estudo que examina a aprendizagem de números decimais usando duas abordagens de ensino distintas: (1) Apresentar soluções incorretas de problemas decimais, de modo que o aprendiz tente identificar, explicar e corrigir o erro; (2) Solicitar que os aprendizes resolvem problemas decimais e recebam feedbacks (abordagem tradicional de ensino).

O objetivo do estudo foi verificar a eficácia do ensino por meio apresentação soluções incorretas, em comparação com metodologia tradicional de resolução de problemas, em que ambos foram incorporados a um sistema tutor online. Assim, o estudo investiga se estudantes expostos a exemplos incorretos podem obter um melhor aproveitamento do que aqueles que resolvem problemas e recebem feedback.

De maneira geral, a prática de identificar, explicar e corrigir erros apresentou trazer benefícios aos aprendizes, sendo que, os submetidos a esta abordagem apresentaram um aprendizado mais aprofundado. Além disso, de acordo com os experimentos realizados, a pesquisa sugere que essa abordagem torna-se mais eficaz com o passar do tempo, e principalmente para aprendizes com pouco conhecimento prévio sobre o assunto tratado. Desse modo, destaca-se que tudo isso também promove evidências para justificar pesquisas em aprendizado a partir dos erros.

Tendo isso em vista, foi proposta uma ferramenta de autoria denominada FARMA ${ }^{4}$ (Ferramenta de Autoria para a Remediação de erros com Mobilidade na Aprendizagem). Ela permite a construção de exercícios voltados ao aprendizado de conceitos de indução analítica que envolvem expressões aritméticas e/ou algébricas. Suas principais características são a de constituir uma ferramenta simples e objetiva para o desenvolvimento de objetos de aprendizagens altamente interativos e promover uma aprendizagem por erros, dos quais são registrados desde a hora em que ocorrem até seus respectivos contextos. Assim, os aprendizes podem, posteriormente, explorar seus próprios erros, além de permitir que os professores tenham acesso integrado ou individual aos

\footnotetext{
${ }^{4}$ Site oficial: farma.educacional.mat.br
} 
aprendizes de uma turma virtual.

\section{Arquitetura Funcionalista}

O objetivo dessa seção é apresentar a arquitetura fun- cionalista da FARMA, que foi desenvolvida para consolidar os fundamentos de aprendizagem descritos na Seção 3. Conforme apresentado na Figura 1, a arquitetura possui três estágios principais: autoria, interação e acompanhamento.

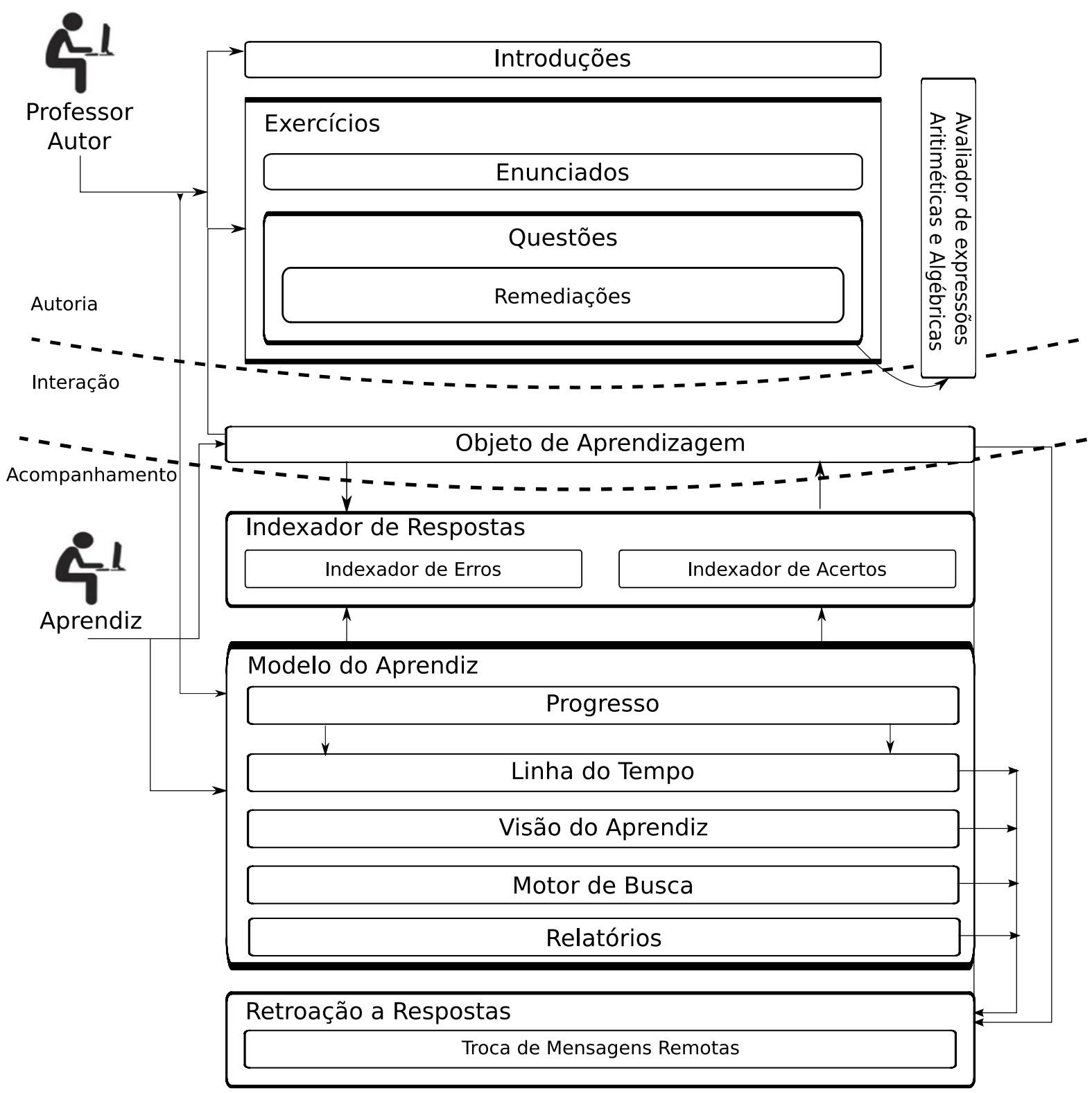

Figura 1: Arquitetura funcionalista da FARMA

A implementação foi realizada visando seu uso na web, seja por meio de computadores pessoais ou de dispositivos móveis. Como plataforma de desenvolvimento, foi utilizada a linguagem Ruby ${ }^{5}$, juntamente com o fra-

${ }^{5}$ Site oficial: https://www.ruby-lang.org mework Ruby on Rails ${ }^{6}$, seguindo as técnicas de Orientação a Objetos e os principais Padrões de Projeto utilizados na web.

A seguir são descritos os três módulos principais da

\footnotetext{
${ }^{6}$ http://rubyonrails.org
} 
arquitetura da FARMA, a saber:

Módulo de Autoria: Fornece todo arcabouço necessário para a construção de objetos de aprendizagem matemáticos. Ele permite a elaboração da parte teórica de um OA, chamada de introduções, e também da parte prática, que são os exercícios.

Módulo de Interação: Interface entre o aprendiz e o OA criado pelo professor-autor. Este módulo realiza a junção das introduções, exercícios, questões e remediações a curto prazo, definidas pelo professor-autor, para compor um OA. A interação do aprendiz com as questões propostas pelo professor-autor é feita por meio de um teclado virtual que se modifica de acordo com a resposta fornecida pelo professor-autor. Por exemplo, se o professorautor definir uma variável na resposta de referência, essa estará disponível no teclado virtual; caso contrário, não existirão variáveis no teclado. Além disso, o teclado virtual faz validação da entrada da resposta do aprendiz, para evitar que seja enviada, como resposta, com expressão matemática mal formada ( $\mathrm{p}$. ex. $\mathrm{x}+/ 2$ ), impedindo que a resposta contenha erros de sintaxe.
Módulo de Acompanhamento: Por meio desse módulo, o professor-autor pode realizar uma avaliação formativa do aprendiz. O principal recurso para isso é o mecanismo de retroação a erros, porque, por meio dele, é possível acessar o contexto exato da ocorrência do erro do aprendiz. Para isso, o modulo subdivide-se em 3 partes principais. São elas: (a) Indexador de respostas, que salva todas as respostas enviadas pelo aprendiz, sejam elas erradas ou corretas. (b) Modelo do aprendiz, que apresenta informações ao professor-autor sobre a interação do aprendiz, como: (1) o progresso do aprendiz; (2) a linha do tempo do aprendiz; (3) a visão do aprendiz sobre o OA e (4) relatórios sobre interação; (c) Retroação a respostas, que permite o professor-autor retroceder ao contexto exato de uma resposta do aprendiz, analisar e promover discussões com o aprendiz sobre a mesma.

\section{Dinâmica do arcabouço}

A dinâmica de funcionamento proposta para esta ferramenta pode ser observada na Figura 2.

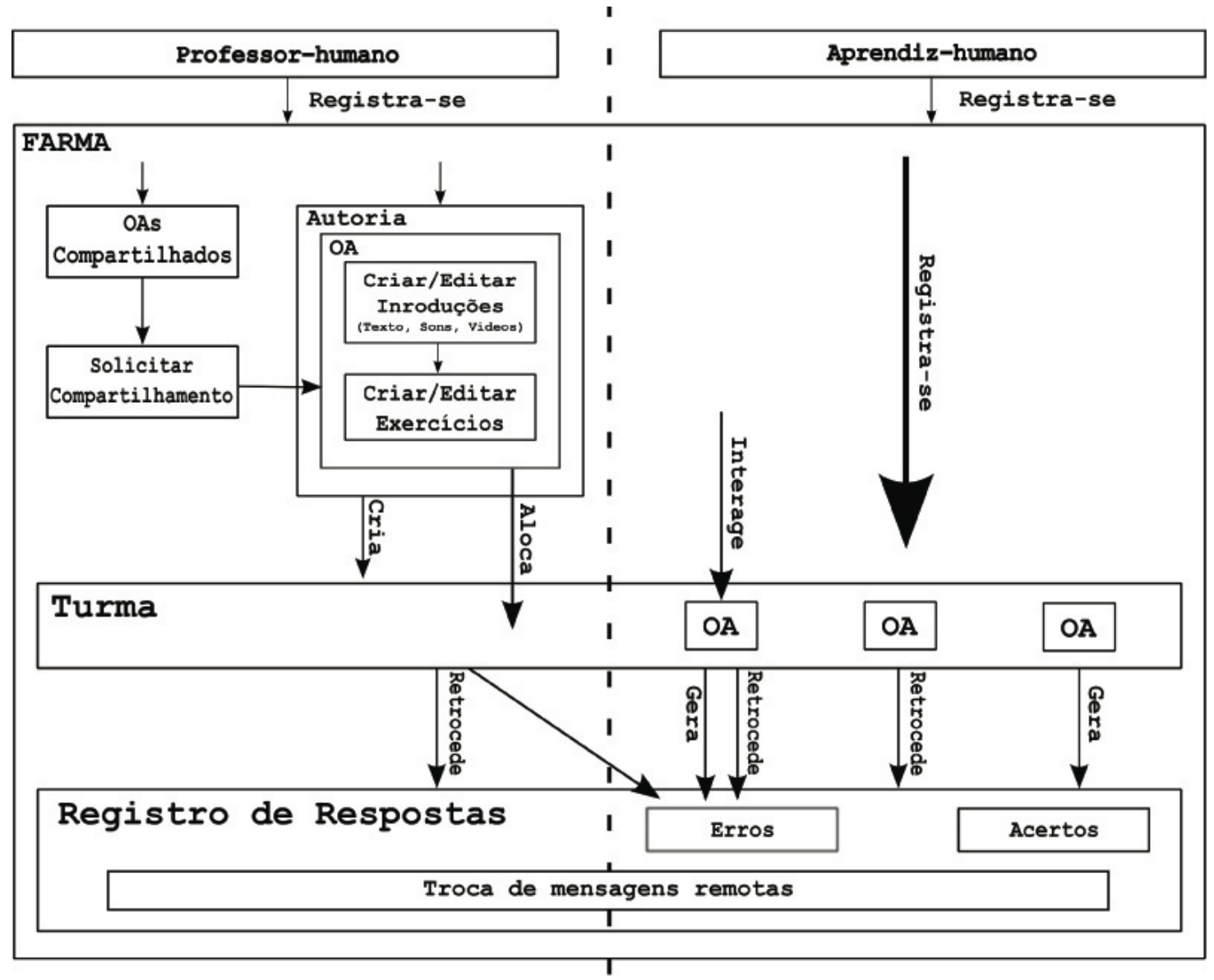

Figura 2: Dinâmica de funcionamento do arcabouço 


\subsection{Esquema Resumido}

$\mathrm{O}$ processo inicia a partir de dois atores principais, $\mathrm{o}$ professor e o aprendiz, os quais primeiramente devem se registrar na ferramenta para posterior interação. Na perspectiva do professor, ele pode começar observando os OAs já desenvolvidos por outros professores e, quando se interessar por algum deles, pode solicitar o compartilhamento e então usá-lo tanto da maneira como o visualizou, como podendo editá-lo conforme necessário. Outro possível caminho que o professor pode seguir é criar seu próprio OA. Um OA, da maneira como foi projetado, será composto por introduções a conteúdos seguidas de exercícios interativos. Para aplicação dos OAs desenvolvidos junto aos aprendizes, o professor primeiramente deve criar uma turma virtual e alocar os objetos para que os aprendizes interajam.

Para os aprendizes interagirem com os OAs, antes devem se matricular em uma turma, geralmente indicada por um professor. Na turma matriculada, estarão todos os OAs vinculados pelo professor. Durante a interação do aprendiz, a ferramenta registra o seu histórico com o OA, incluindo seções de estudo em que houve envio de respostas corretas e incorretas. Posteriormente, tanto o professor quanto o aprendiz podem retroceder aos erros cometidos e podem também tentar refazer os exercícios a partir do seu erro. Durante esse processo, também é possível a discussão entre professores e aprendizes sobre suas respostas, principalmente sobre seus erros.

\subsection{Construção do OA}

$\mathrm{O}$ arcabouço proposto visa à construção de OAs compostos por introduções a conteúdos seguidos de exercícios no formato de uma sequência de páginas. Este formato tem por objetivo colocar em cada página pequenas partes do conteúdo para facilitar a concentração do aprendiz em uma sequência de soluções restritas a um enunciado comum.

Para a utilização dos OAs criados, assim como para a própria criação não é necessário qualquer tipo de instalação adicional, apenas um dispositivo, seja um computador desktop, ou um aparelho móvel que possua um navegador (p. ex. Firefox ou Chrome) com acesso à Internet. Toda interação do professor e do aprendiz é feita online, sendo que o professor pode até acompanhar a interação do aprendiz com o conteúdo criado, visualizando suas respostas em tempo real.

Para a criação das introduções (parte teórica de um OA criado com a FARMA), o professor pode usufruir dos mais variados formatos de conteúdo como texto, imagens, sons, vídeos entre outros tipos que possam ser in- corporados no formato HTML. Junto do CSS, ambos são frequentemente usados para compor o design das páginas criadas durante a elaboração do OA. Para isso, não é exigido nenhum conhecimento adicional sobre esse assunto por parte do professor-autor, pois todo o processo de elaboração é feito por meio de interfaces WYSI$\mathrm{WYG}^{7}$.

Para os enunciados dos exercícios e das questões, os mesmos formatos são permitidos. No arcabouço proposto, o exercício contém um título e um enunciado. Então ele é subdividido em vários outros tópicos, chamados de questões. Cada questão, além do enunciado, contém um campo para a entrada da resposta correta, que é definida por meio de uma expressão algébrica ou aritmética no formato de texto, seguindo os padrões de escrita convencionais da Matemática.

Outro fator importante para as questões é fornecer mecanismos para que o professor permita que o aprendiz se recupere de ações repetidas sem sucesso durante a resolução de uma questão. Uma das maneiras de alcançar esse objetivo é fornecer dicas (p. ex. textos, imagens, etc.) sobre as respostas sem sucesso do aprendiz. Isso caracteriza-se como uma forma de remediação de erros, que viabiliza a intervenção junto ao aprendiz antes da progressão em um determinado erro, evitando, assim, uma solução completa, mas equivocada.

A divisão dos exercícios em etapas de solução segue a ideia da técnica model tracing da Teoria ACT, isso promove o desenvolvimento gradual da capacidade de abstração do aprendiz. Para ficar mais claro, observe o enunciado a seguir:

Um ciclista acrobático deseja atravessar de um prédio ao outro com uma bicicleta especial, por meio de um cabo de aço rígido. $\mathrm{O}$ prédio de partida tem a altura de 75 metros e o de chegada tem a altura de 25 metros. A distância entre as duas torres é de 120 metros.

Qual é o comprimento do cabo de aço que vai de um prédio a outro?

Apresentando somente esse enunciado ao aprendiz, ele teria de abstrair todas as etapas de resolução sozinho, sendo exigida uma maior capacidade de abstração do mesmo. Tal fato pode ser destacado como uma dificuldade frequente dos alunos, ainda mais quando ocorre a apresentação de um novo conceito.

Em contrapartida, o mesmo exercício, pode ser dividido em etapas, promovendo o acompanhamento do caminho de solução do aluno e assim permitindo a apresentação de uma remediação (a curto prazo) mais precisa, no caso de equívoco cometido pelo aprendiz. Assim esse

${ }^{7}$ What You See Is What You Get. 
enunciado poderia ser exibido em várias etapas conforme apresentado a seguir:

\section{Enunciado 1}

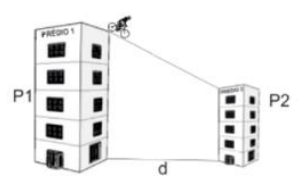

Um ciclista acrobático deseja atravessar de um prédio ao outro com uma bicicleta especial, por meio de um cabo de aço rígido. $O$ prédio de partida tem a altura de 75 metros e o de chegada tem a altura de 25 metros. A distância entre as duas torres é de 120 metros.

\section{Etapa 1}

Quais as medidas indicadas na figura? Resposta: 75; 25; 120.

Caso o aprendiz insira uma resposta incorreta para essa questão as seguintes remediações (a curto prazo), de acordo com o número de tentativas, poderiam ser apresentadas, sendo uma a cada erro seguindo a ordem:

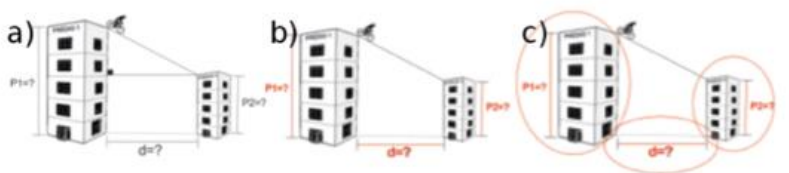

\section{Etapa 2}

Qual a diferença de altura entre as duas torres? Resposta: 50 .

Possíveis remediações: a)

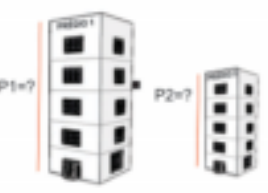

b)

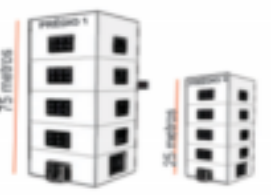

\section{Etapa 3}

Para descobrir o comprimento mínimo do cabo de aço que liga as duas torres é necessário extrair um triângulo retângulo da figura citada e então aplicar o Teorema de Pitágoras. Assim, quais seriam as medidas dos catetos do triângulo? Resposta: 50; 120.

\section{Possíveis remediações:}

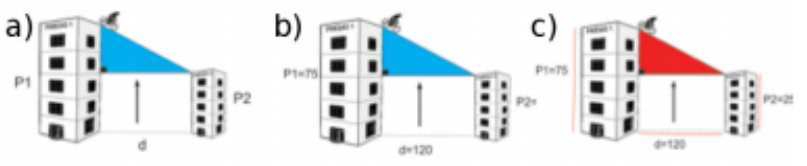

\section{Etapa 4}

Sabendo que o comprimento do cabo de aço é representado pela letra 'a', apresente a equação do Teorema de Pitágoras aplicada a figura do ciclista para descobrir o comprimento mínimo necessário do cabo de aço para ir de uma torre a outra. Resposta: $\mathrm{a}^{\wedge} 2=50^{\wedge} 2+120^{\wedge} 2$.

Possíveis remediações: a)

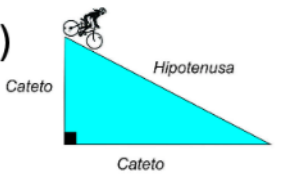

(hipotenusa $)^{2}=(\text { cateto })^{2}+(\text { cateto })^{2}$ b)

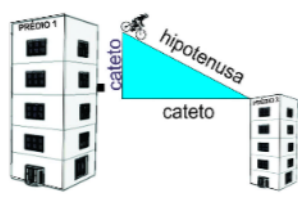

\section{Etapa 5}

Qual é o comprimento mínimo do cabo de aço necessário para ir de um prédio ao outro? Resposta: 130.

Possíveis remediações:

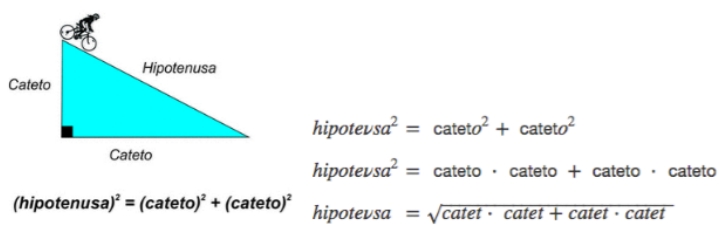

Seguindo essa abordagem é possível oferecer um aprendizado gradual por meio da estruturação de passos (transformados em etapas no OA), amparados pelos conceitos do model tracing da Teoria ACT. Sendo que a cada erro cometido pelo aprendiz é apresentado uma dica apropriada, de acordo com o número de tentativas.

\section{Avaliação Experimental da FARMA}

Este seção apresenta os experimentos realizados com o intuito de avaliar a ferramenta de autoria FARMA, tanto para a criação e aplicação de OAs quanto à utilização do mecanismo de retroação a contexto de erros. Para isso, foram realizados estudos empíricos e análises estatísticas, porém neste artigo será abordado apenas as análises estatística de um dos experimentos realizados.

\subsection{Avaliação Experimental do Mecanismo de Retroação}

Este experimento tem o intuito de avaliar formalmente o mecanismo de retroação a erros como recurso adicional para aquisição de conhecimento do aprendiz. Para isso, foi elaborado um experimento e consequente análise dos dados por meio do método estatístico test- $t$ e posteri- 
ormente pelo teste não paramétrico Wilcoxon-MannWhitney.

Para o experimento foi escolhido o tema Logaritmos, conteúdo regular da matriz curricular das turmas participantes. Destaca-se que esse conteúdo estava sendo normalmente ministrado pelo professor de Matemática das turmas participantes do experimento, aplicando assim a ferramenta em um ambiente real de estudo.

\subsubsection{Sujeitos}

Os sujeitos deste experimento foram os alunos de 3 turmas da disciplina de Matemática do Centro Estadual de Educação Profissional de Curitiba, contando inicialmente com um total de 70 alunos divididos em dois grupos: Grupo Controle (GC) e Grupo Experimental (GE). Os participantes do experimento foram informados sobre os objetivos, bem como do caráter voluntário e sigiloso da sua participação.

\subsubsection{Formação dos Grupos}

Como o objetivo do teste foi comparar 2 (dois) grupos, os alunos das 3 (três) turmas foram divididos em duas partes a partir do resultado da aplicação de um préteste.

O pré-teste foi composto por 10 questões desenvolvidas pelo professor da disciplina e aplicado como parte da avaliação dos aprendizes. Pelas notas obtidas por cada aprendiz foram definidos os participantes de cada grupo, GC e GE. A fim de tornar os grupos homogêneos, os aprendizes foram ordenados de acordo com suas notas e então foi realizada divisão em duas turmas, por meio de uma seleção por pareamento e sorteio quando notas iguais.

Grupo de Controle: composto por 35 alunos, presenciou aulas regulares da disciplina de Matemática sobre o assunto de logaritmos e utilizou o OA desenvolvido sem o mecanismo de retroação.

Grupo de Experimental: composto por 35 alunos, presenciou aulas regulares da disciplina de Matemática sobre o assunto de logaritmos e utilizou o OA desenvolvido com o mecanismo de retroação.

Salienta-se que os grupos GC e GE foram compostos por alunos provenientes das 3 (três) turmas. Assim tevese o cuidado de promover um tratamento imparcial quanto ao conteúdo ministrado, a carga horária letiva e também sobre as aplicações do pré e pós-teste.

\subsubsection{Metodologia}

O experimento realizado teve três etapas principais:

1. Pré-teste;

2. Interação com o OA Logaritmos;

3. Pós-teste.

Como o objetivo principal era avaliar a contribuição do uso do mecanismo de retroação no processo de ensino e aprendizagem, primeiramente foi elaborado pelo professor da disciplina uma prova (usada como pré-teste) envolvendo os conceitos iniciais de logaritmos já estudados na disciplina. O resultado foi utilizado para identificar o conhecimento inicial dos aprendizes. Então, o professor da disciplina desenvolveu um OA para o ensino de conceitos de logaritmos utilizando a FARMA. Posteriormente, o OA foi utilizado pelos membros dos dois grupos (GC e GE). Finalmente, para verificar o ganho na aprendizagem, foi aplicado um pós-teste, elaborado pelo professor da disciplina e aplicado como uma prova pertencente aos métodos de avaliação da disciplina.

O objetivo do pré-teste é inicialmente definir os participantes de cada grupo (GC e GE) e, posteriormente, ser usado como comparativo com o resultado do pósteste. O pré-teste foi composto por 10 questões, que envolvem conceitos relacionados à área de logaritmos, onde o número de acertos foi norteador a fim de tornar os grupos homogêneos.

O experimento foi realizado em 3 etapas principais. $\mathrm{Na}$ primeira etapa foi aplicado o pré-teste objetivando identificar os conhecimentos prévios em relação ao conteúdo abordado e definir a distribuição dos grupos.

$\mathrm{Na}$ aula seguinte à aplicação do pré-teste, os participantes foram incentivados a interagirem com o OA proposto. Destaca-se que o professor também foi incentivado a interagir com o mecanismo de retroação para verificar o andamento da turma sobre as atividades do OA.

A interação dos aprendizes com o OA foi realizada fora do horário normal de aula. Desse modo, os aprendizes interagiram com o OA em suas casas ou no laboratório da escola em horário extra-classe. Devido à retroação de erros poder ser usada a longo prazo, foi determinado um tempo de 3 (três) semanas para os aprendizes interagirem com o OA e resolverem todas as questões propostas.

Posteriormente a esse prazo, foi realizada a aplicação do pós-teste, composto por 12 questões, divididas em 4 (quatro) exercícios que envolvem novamente conceitos relacionados a logaritmo estudados em sala e no OA, mas distintas das questões aplicadas no pré-teste, embora com 
o mesmo grau de dificuldade. O objetivo do pós-teste é confrontar os resultados para perceber a evolução na aprendizagem dos alunos, que interagiram com os Objetos de Aprendizagem.

\subsubsection{O OA Logaritmos}

O OA Logaritmos envolve temas sobre: (1) História dos logaritmos; (2) O conceito de logaritmo; (3) Exemplo de uso; (4) Logaritmo decimal; (5) Propriedades dos logaritmos; (6) Equações logarítmicas; (7) Logaritmos e terremotos.

Composto por 35 questões divididas em 5 exercícios. Outro ponto de destaque é que o OA fornece um aprendizado gradual por meio da estruturação de passos, na qual as atividades foram transformadas em etapas de resolução a partir do enunciado do problema, possibilitando aplicar os conceitos presentes no ACT, como por exemplo o model tracing.

\section{Resultados}

Após a aplicação do OA, foi realizada uma análise da interação do aprendiz. Nela surgiram evidências de compartilhamento de login e senha e cópias de respostas entre os aprendizes. Então, investigou-se os aprendizes que tiveram indícios de cópia de respostas e estes foram excluídos do experimento. Para exemplificar, houve casos em que o aprendiz resolveu as 35 questões do OA em menos de 15 minutos, sendo que teve apenas 1 erro em uma das questões, isso na noite antes do prazo final de resolução. Outros casos, mostram que o aprendiz resolveu várias questões com um intervalo médio de 15 segundos entre cada questão, isso ainda sem errar. Diante disso, ressalta-se que esses fatos não são factíveis com a realidade proposta de resolução.

Aprendizes, com esses tipos de comportamento independente da nota do pré-teste e pós-teste foram excluídos do experimento. Com isso, 27 aprendizes tiveram de ser excluídos do experimento, ficando com 24 aprendizes que conduziram o experimento de maneira séria.

Assim, cada grupo ficou com 12 aprendizes, as tabelas 1 e 2 apresentam as notas do pré-teste e pós-teste, média e desvio padrão desses aprendizes, além da porcentagem de conclusão do $\mathrm{OA}$; e, para o $\mathrm{GE}$, o número de vezes que utilizou o mecanismo de retroação.

\begin{tabular}{|c|c|c|c|}
\hline \multicolumn{5}{|c|}{ Grupo Controle } \\
\hline Aprendiz & Pré-teste & Completude & Pós-teste \\
\hline 01 & 1,0 & $100 \%$ & 0,77 \\
\hline 02 & 0,9 & $100 \%$ & 0,83 \\
\hline 03 & 0,6 & $97,14 \%$ & 0,37 \\
\hline 04 & 0,5 & $100 \%$ & 0,53 \\
\hline 05 & 0,5 & $82,85 \%$ & 0,53 \\
\hline 06 & 0,5 & $82,85 \%$ & 0,47 \\
\hline 07 & 0,2 & $100 \%$ & 0,48 \\
\hline 08 & 1,0 & $100 \%$ & 0,67 \\
\hline 09 & 0,5 & $31,42 \%$ & 0,43 \\
\hline 10 & 0,5 & $82,85 \%$ & 0,40 \\
\hline 11 & 0,8 & $62,85 \%$ & 0,53 \\
\hline 12 & 0,6 & $42,85 \%$ & 0,67 \\
\hline Mediana & $\mathbf{0 , 5 5}$ & $\mathbf{9 0 \%}$ & $\mathbf{0 , 5 3}$ \\
\hline Média & $\mathbf{0 , 6 3}$ & $\mathbf{8 1 , 9 0 \%}$ & $\mathbf{0 , 5 5}$ \\
\hline Desvio Padrão & $\mathbf{0 , 2 4}$ & $\mathbf{2 3 , 9 4}$ & $\mathbf{0 , 1 4}$ \\
\hline
\end{tabular}

Tabela 1: Dados do Grupo Controle

\begin{tabular}{|c|c|c|c|c|}
\hline \multicolumn{5}{|c|}{ Grupo Experimental } \\
\hline Aprendiz & Pré-teste & Completude & Pós-teste & $\begin{array}{c}\text { Uso da } \\
\text { retroação }\end{array}$ \\
\hline 01 & 1,0 & $100 \%$ & 0,7 & 4 \\
\hline 02 & 0,8 & $100 \%$ & 0,8 & 3 \\
\hline 03 & 0,8 & $88,57 \%$ & 0,9 & 2 \\
\hline 04 & 0,6 & $100 \%$ & 0,73 & 3 \\
\hline 05 & 0,5 & $97,14 \%$ & 0,67 & 58 \\
\hline 06 & 0,3 & $22,85 \%$ & 0,3 & 3 \\
\hline 07 & 0,9 & $94,28 \%$ & 0,8 & 44 \\
\hline 08 & 0,9 & $97,14 \%$ & 0,87 & 6 \\
\hline 09 & 0,8 & $82,85 \%$ & 0,8 & 1 \\
\hline 10 & 0,5 & $74,28 \%$ & 0,53 & 13 \\
\hline 11 & 0,3 & $100 \%$ & 0,53 & 22 \\
\hline 12 & 0,7 & $100 \%$ & 0,67 & 1 \\
\hline Mediana & 0,7500 & $97,14 \%$ & 0,7150 & 3,500 \\
\hline Média & 0,6750 & $88,09 \%$ & 0,6917 & 13,33 \\
\hline $\begin{array}{c}\text { Desvio } \\
\text { Padrão }\end{array}$ & 0,2340 & $22,13 \%$ & 0,17018 & 18,84064 \\
\hline
\end{tabular}

Tabela 2: Dados do Grupo Experimental

Um dos fatores que pode ter contribuído para a contaminação do experimento, foi a possibilidade dos aprendizes realizarem as atividades em casa e nos laboratórios da escola sem a presença do professor. Assim, destacamse como principais causas que podem ter afetado o experimento:

- Falta de controle sobre a forma que os aprendizes usaram a ferramenta e o OA;

- Falta de flexibilidade, pois não foi possível realizar uma estratégia para acompanhar os alunos durante a realização do experimento;

- Não disponibilização de aulas exclusivas para interação dos aprendizes com o $\mathrm{OA}$;

- Falta de seriedade de vários aprendizes, principal- 
mente por aqueles que realizaram cópias das atividades propostas.

Considerando o fato de os tamanhos finais das amostras dos grupos (após o tratamento) serem relativamente pequenos (12 elementos para cada grupo) para a aplicação do teste paramétrico test-t (t-Student), optou-se então pelo uso do teste estatístico não paramétrico WilcoxonMann-Whitney, considerado pelos estatísticos um dos testes mais poderosos e em alguns casos, supera o poder do test- $t$. Sendo que possui uma maior eficiência que o test- $t$ para distribuições não normais e é considerado quase tão eficaz como o test-t para distribuições normais [20].

No gráfico da Figura 3, tem-se a comparação entre as amostras Controle e Experimental. Destaca-se que as diferenças entre os pré-testes dos dois Grupos não foram significantes (GE com média de 0,6750 e o GC com média de 0,6333 , com $\mathrm{W}=64,5$ e p-valor atribuído $=$ $0,6809)$, indicando que os grupos possuem mesmo nível de conhecimento. Na verificação entre os pós-testes, houve uma diferença significativa, que também pode ser observada no gráfico. Isso comprova que as notas do pósteste do GE (média de 0,6917) foram melhores que as notas do pós-teste do GC (média de 0,5567) com $\mathrm{W}=$ $37,5, \mathbf{p}$-valor atribuído $=0,0483$.
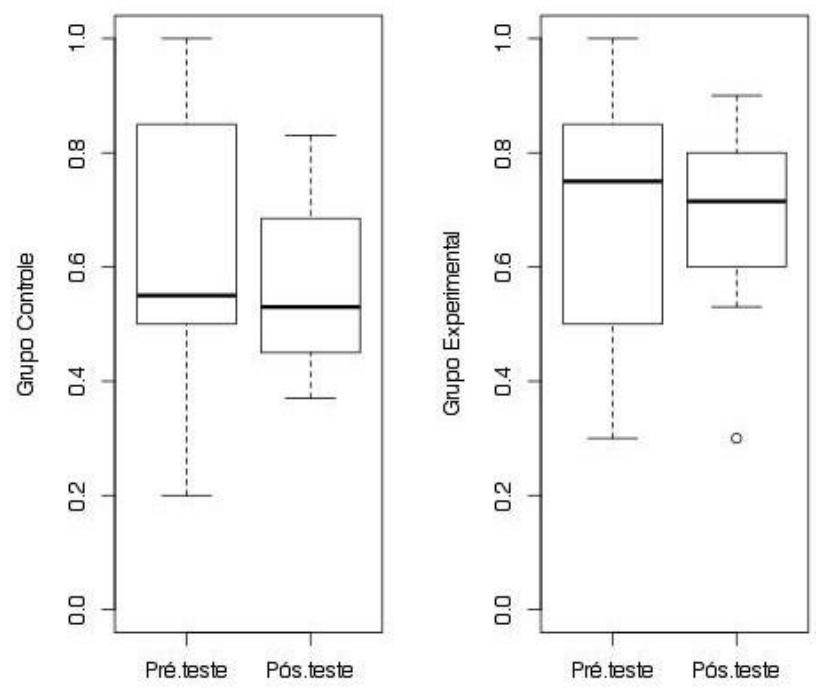

Figure 1: Comparação entre as amostras Controle e Experimental

No gráfico da Figura 3, tem-se a comparação entre as amostras GC e GE. Destaca-se que as diferenças entre os pré-testes dos dois Grupos não foram significantes , indicando que os grupos possuem mesmo nível de conhecimento.

$\mathrm{Na}$ verificação entre os pós-testes houve uma diferen- ça significativa, que também pode ser observada no gráfico. Isso comprova que as notas do pós-teste do GE foram melhores que as notas do pós-teste do GC.

Apesar do resultado favorável para o GE, evidenciase que o estudo apresenta certas tendências que podem indicar os chamados efeitos de bordura. Em experimentação, diz-se efeito de bordura quando uma parcela de tratamento, ou elemento da amostra influencia outra, quando na verdade deveria existir independência entre eles. No caso de aplicação do teste, o fato de trabalhar com o OA em um ambiente livre, pode ter comprometido os resultados, por isso, as conclusões, ainda que favoráveis, podem ser superficiais. Assim, destaca-se a importância de um novo experimento em que se possa garantir o máximo do tamanho da amostra submetido à pesquisa, e ainda sem a influência de fatores que possam comprometer os dados.

\section{Considerações Finais e Trabalhos Futuros}

Essa pesquisa destaca a manipulação dos registros de erros, por meio da retroação ao contexto de erros e acertos acoplado a uma ferramenta de autoria web. Desse modo, argumenta-se que o armazenamento, a visualização, a manipulação e a recuperação de registros de erros e acertos dos aprendizes podem trazer benefícios para o ensino e aprendizagem.

Acredita-se que esta pesquisa abre espaço para que sejam retomados os estudos sobre uso do erro como fonte de oportunidade para o ensino e aprendizagem, principalmente em uma nova perspectiva proposta, a retroação a erros. Isso com o intuito que ele seja visto com uma oportunidade e uma fonte de informações tanto para o aprendiz quanto para o professor. Para o aprendiz, de modo que ele possa refletir e se recuperar do erro cometido e, para o professor, como fonte de dados para fornecer feedbacks mais precisos.

Ainda que apresentada essa abordagem, são necessárias pesquisas com o intuito de determinar o melhor momento e a melhor forma de apresentar a retroação a erros ao aprendiz. Do mesmo modo, são necessárias pesquisas para que o professor não precise revisar todos os erros de uma turma ou grupo de aprendizes, o que se tornaria impraticável, quando o número de alunos de uma turma é grande. Mas sim, que, a própria FARMA proporcione, por meio das similaridades dos erros, grupos de erros para o professor analisar, e então replique a análise, de maneira automática, para os demais erros, como proposto 
por Kutzke [21] para área de programação de computadores, só que para conceitos matemáticos.

Outros trabalhos futuros apontam para pesquisas relacionadas à mineração dos erros cometidos pelo aprendiz, de modo a fornecer informações mais precisas ao professor sobre as dificuldades dos aprendizes. Adicionalmente, destaca-se a junção das avaliações formativa, diagnóstica automática e somativa em uma única ferramenta, a FARMA. De modo que, por meio da aplicação de um OA construído com a FARMA, seja possível que o professor tenha acesso essas três avaliações.

Assim, pode-se consolidar um elenco de medidas cognitivas no aprendizado da Matemática (não exclusivamente), sob a perspectiva dos erros por meio da FARMA e seu mecanismo de retroação. Trata-se de uma linha de pesquisa promissora visto que os resultados obtidos podem potencializar o tempo e esforço de professores e aprendizes.

A contribuição se estende também no sentido de proporcionar o uso de estratégias de ensino por meio de recursos tecnológicos, mais eficientes. Que considera o erro do aprendiz como uma etapa natural no processo de aprendizagem, seja ele embutido em recursos tecnológicos ou em sessões convencionais de ensino.

Por fim, destaque-se que a FARMA saiu do laboratório de pesquisa e está sendo usada em ambientes reais de ensino, constituindo assim uma contribuição relevante para a área de Informática Aplicada à Educação.

\section{Referências}

[1] M. L. P. Flores. Metodologia para criar Objetos de Aprendizagem em matemática usando a combinação de ferramentas de autoria. Tese de doutorado, Universidade Federal do Rio Grande do Sul - UFRS, 2011.

[2] M. R. Notare e P. A. Behar. Comunicação e aprendizagem matemática on-line: Um estudo com o editor científico rooda exata. Revista Brasileira de Informática na Educação, 18(1), 2010.

[3] T. Murray, S. Blessing e S. Ainsworth. Authoring tools for advanced technology learning environments: Toward cost-effective adaptive, interactive and intelligent educational software. Springer, 2003.
[4] M. L. P. Flores. Metodologia para criar Objetos de Aprendizagem em matemática usando a combinação de ferramentas de autoria. Tese de doutorado, Universidade Federal do Rio Grande do Sul - UFRS, 2011.

[5] A. Direne, W. Silva, F. Silva, L. Peres, A. Kutzke, D. Marczal, G. Barros, L. Moura e G. Bazzo. Aprofundamento da mobilidade tecnológicoeducacional por meio de jogos intelectivos. In Anais do DesafIE-2012, pages 1-10. SBC, 2012.

[6] T. Murray, B. Woolf e D. Marshall. Lessons learned from authoring for inquiry learning: A tale of authoring tool evolution. In Proc. of the 7th International Conference on Intelligent $\mathrm{Tu}-$ toring Systems (ITS2004), pages 782-784. Springer, 2004.

[7] S. Ritter, J. R. Anderson, K. R. Koedinger e A. Corbett. Cognitive tutor: Applied research in mathematics education. Psychonomic bulletin \& review, 14(2):249-255, 2007.

[8] H. Seffrin, G. Rubi, C. Ghilardi, F. Morais, P. Jaques, S. Isotani e I. Bittencourt. Dicas inteligentes no sistema tutor inteligente PAT2Math. In Anais do Simpósio Brasileiro de Informática na Educação, volume 23, 2012.

[9] R. Z. Cabada, M. L. B. Estrada e C. A. R. García. Educa: A web 2.0 authoring tool for developing adaptive and intelligent tutoring systems using a Kohonen network. Expert Systems with Applications, 38(8):9522 - 9529, 2011.

[10] V. Aleven e J. Sewall. Hands-on introduction to creating intelligent tutoring systems without programming using the cognitive tutor authoring tools (CTAT). In Proceedings of the 9th International Conference of the Learning SciencesVolume 2, pages 511-512, 2010.

[11] S. Isotani e L. D. O. Brandão. Ferramenta de avaliação automática no iGeom. In Anais do Simpósio Brasileiro de Informática na Educação, volume 1, páginas 319-328, 2004.

[12] P. E. Battistella e A. Von Wangenheim. Avaliação de ferramentas de autoria gratuitas para produção de Objetos de Aprendizagem no padrão SCORM. Revista Brasileira de Informática na Educação, 19(3):16-28, 2011.

[13] J. W. Stamey e B. Saunders. Designing intelligent learning objects. In Advanced Learning Technologies, 2005. ICALT 2005. Fifth IEEE International Conference on, pages 323-325, 2005. 
[14] L. S. Vygotski. A formação social da mente. Psicologia, 153:V631, 1989.

[15] M. D. Leite, A. R. Pimentel, and M. H. Pietruchinski. Remediação de erros baseada em múltiplas representações externas e classificação de erros aplicada a Objetos de Aprendizagem inteligentes. In Anais do Simpósio Brasileiro de Informática na Educação, volume 23, 2012.

[16] A. Peng and Z. Luo. A framework for examining mathematics teacher knowledge as used in error analysis. For the learning of mathematics, pages 22-25, 2009a.

[17] Seiji Isotani, Deanne Adams, Richard E Mayer, Kelley Durkin, Bethany Rittle-Johnson, and Bruce M McLaren. Can erroneous examples help middle-school students learn decimals? In Towards Ubiquitous Learning, pages 181-195. Springer BerlinHeidelberg, Berlin, Heidelberg, 2011.

[18] Bruce M McLaren, Deanne Adams, Kelley Durkin, George Goguadze, Richard E Mayer, Bethany Rittle-Johnson, Sergey Sosnovsky, Seiji Isotani, and Martin Van Velsen. To err is human, to explain and correct is divine: a study of interactive erroneous examples 124 with middle school math students. In 21st Century Learning for 21st Century Skills, pages 222-235. Springer, 2012.

[19] Adams, D. M., McLaren, B. M., Durkin, K., Mayer, R. E., Rittle-Johnson, B., Isotani, S., et al. (2014). Using erroneous examples to improve mathematics learning with a web-based tutoring system. Computers in Human Behavior, 36, 401-411.

[20] Sidney Siegel. Estatística não-paramétrica para as ciências do comportamento. McGraw- Hill São Paulo, 1975.

[21] A. R. Kutzke. Informática educacional e a mediação do erro na educação: um estudo teóricocrítico e uma proposta de instrumento computacional. Tese de doutorado, Universidade Federal do Paraná, UFPR, 2015.
Artigo referente à seguinte tese, que obteve primeiro lugar no Concurso de Teses, Dissertações e Trabalhos de Conclusão de Curso em Informática na Educação (CTDIE) de 2015:

D. Marczal. FARMA: Uma Ferramenta de Autoria para Objetos de Aprendizagem de Conceitos Matemáticos. Tese de doutorado, Universidade Federal do Paraná, Novembro 2014. 